\title{
THE CARBOHYDRATES IN NORMAL AND ABNORMAL CEREBROSPINAL FLUID
}

\author{
BY
}

\author{
MARJORIE D. EASTHAM AND KENNETH R. KEAY \\ From the Department of Biochemistry, University College, Dundee, and the Department of Paediatrics. \\ the Medical School, University of St. Andrews
}

(RECEIVED FOR PUBLICATION APRIL 8, 1952)

Determination of the sugar content forms an essential part in the complete examination of cerebrospinal fluid. The sugar determined is generally considered to be mainly glucose (Harrison, 1947).

Hubbard and Russell (1937), using a resorcinol colorimetric estimation, found a ketose in varying amounts which they thought was probably fructose.

In the present study a discrepancy was observed between the value of reducing compounds determined in cerebrospinal fluid by the method of Hagedorn and Jensen and that of Somogyi (1945) comparable to that observed by Somogyi and Kramer (1928) in blood sugar determination. It was believed that paper partition chromatography might throw some light on the reasons for this discrepancy. A comparative study has been made in 100 cerebrospinal fluids using four different methods of examination for reducing sugars: (1) Benedict's test; (2) the Hagedorn-Jensen estimation ; (3) Somogyi copper-phosphate estimation ; (4) paper partition chromatography, qualitative (Partridge, 1948) and quantitative (Flood, Hirst, and Jones, 1947).

It is generally agreed that the various forms of meningitis are accompanied by a fall in the amount of reducing substance in cerebrospinal fluid below $45 \mathrm{mg}$. $/ 100 \mathrm{ml}$. (MacCarthy and Mann, 1950). In certain cases of meningitis, however, values greater than this have been reported. With the advent of streptomycin treatment for tuberculous meningitis early diagnosis is of first importance, and it seemed useful to know (1) if the discrepancy between methods was extensive amongst normals and existed also in the anomalous meningitis cases, and (2) what was the nature of the reducing substance.

\section{Methods}

Preservation of Specimens.-In accordance with the work of Cohen (1936), whose experimental results on preservation were first confirmed on 17 samples, specimens were collected under sterile conditions and retained at $0^{\circ} \mathrm{C}$. Occasional specimens contained sodium fluoride, which was found to be neither detrimental nor necessary. Specimens were obtained mainly by lumbar puncture; a few from cisternal and lumbar punctures were compared.

Hagedorn-Jensen and Benedict Methods.-These were carried out according to Harrison (1947).

Somogyi Method.-This method (Somogyi, 1945) was carried out using the iodate-iodide titration method.

Qualitative Chromatography. - In this the method finally used was as follows: $2 \mathrm{ml}$. of sample was evaporated in a 1-in. diameter glass dish to a paste on the water bath at $45^{\circ} \mathrm{C}$. and then completely dried in a vacuum desiccator. The desiccate was well mixed with $0.2 \mathrm{ml}$. of methanol and the resulting suspension removed into a $2 \times 0.3 \mathrm{~cm}$. agglutination tube. The salt and protein precipitate was allowed to settle into the pointed end overnight at $0^{\circ} \mathrm{C}$., this period ensuring the solubility of less soluble sugar amines.

A measured volume of $0.05 \mathrm{ml}$. of the clear supernatant methanol extract of the sugars was placed on spots in triplicate on No. 3 Whatman paper, paired alongside mixtures of standard sugars.

After irrigation with butanol-acetic acid for $\mathbf{4 8}$ to $\mathbf{6 0}$ hours the paper was cut vertically into three and the portions sprayed with the three colour reagents: (1) benzidine (Horrocks, 1949) ; (2) naphthoresorcinol and trichloracetic acid at $90^{\circ}-100^{\circ} \mathrm{C}$. in steam (Partridge. 1948 ; Forsyth, 1948); and (3) dimethylaminobenzaldehyde and acetylacetone (Morgan and Elson, 1934) in order to differentiate between aldehyde and ketone sugars, sugar amines, and uronic acids.

Grouping of Cases.-The cases were grouped as (I) " normal"; (II) tuberculous and non-tuberculous meningitis; and (III) miscellaneous cases of disease of the central nervous system. Group III will not be discussed in this paper.

I: "Normals." - This group consisted of 50 cases on which the chromatographic, Hagedorn-Jensen, and Benedict methods were all carried out; on 26 of these 50 cases the Somogyi method was also used.

This group included all cases of specimens collected before spinal anaesthesia, together with those of noncerebrospinal disease in live persons which were normal as regards cell count. protein, Lange gold curve, and "sugar." 
It included also a further 17 cases used for fructose estimation by Roe's method (1934) and 10 cases used for glucosamine estimation by the method of Morgan and Elson (1934).

In both the Roe, and Morgan and Elson, colorimetric methods an E.E.L. photoelectric colorimeter with a green filter was used for the measurements. Reagent blanks and a suitable range of standards were made afresh with each set of readings. Analar standard $\mathrm{HCl}$ and recently opened or redistilled alcohol were used. Test-tubes of $15 \times 150 \mathrm{~mm}$. were used uncovered during heating.

For quantitative chromatography, as described by Flood et al., bulked samples of $30 \mathrm{ml}$. were collected and evaporated and the methanol extract used. Each individual sugar was extracted from its horizontal paper strip with methanol and evaporated for identification.

Quantitative estimations showed little of interest except that there was a zone at $\mathrm{Rf} 0.28-0.30$ containing material which reduced both Hagedorn-Jensen and Somogyi reagents but gave no brown spot with benzidine. showing only a white cone. It was not identified.

Crystals from each strip were examined microscopically. From strip Rf 0.14-0.16 crystalline plates were obtained from concentrated $\mathrm{HCl}$ with a melting point at $142-6^{\circ} \mathrm{C}$. Both phenylhydrazone and osazone were prepared on the microscope slide and compared with those from B.D.H. dextrose. The fructose strip at $\operatorname{Rf} 0.23$ yielded a similar osazone and a methylphenylhydrazine compound of melting point $158^{\circ} \mathrm{C}$.

A further bulked sample of approximately $100 \mathrm{ml}$. was evaporated as collected and retained at $0^{\circ} \mathrm{C}$. for preparation of glucosamine nitrobenzylidene compound by the method of Jolles and Morgan (1940). It yielded crystals like Christmas trees, with a strong hyacinth odour at a melting point of $180-182^{\circ} \mathrm{C}$.. $6 \mathrm{mg}$. in weight, decomposing above this temperature to a brown mass and a white sublimate, similar to those from glucosamine hydrochloride.

The use of bulked samples is open to criticism but individual specimens were chomatographed to ensure normality.

II : Abnormals.-This group includes all the cases where meningeal infection was diagnosed. It has been subdivided into a main group of tuberculous meningitis (IIa) and non-tuberculous meningitis (IIb). Cases of miliary tuberculosis have been included with IIa for purposes of comparison and contrast.

As with the normals in Group I, it was possible to test only a proportion of cases by the Somogyi method owing to insufficiency of the sample, but all were examined by chromatography and the HagedornJensen method and a number by the fructose estimation of Roe.

\section{Results}

Group I.-Fig. 1 shows the subgroup of 26 normals. The Benedict reactions were all red or orange. Somogyi's estimations vary between 35 and $90 \mathrm{mg}$. with an average of $67 \mathrm{mg} . / 100 \mathrm{ml}$.

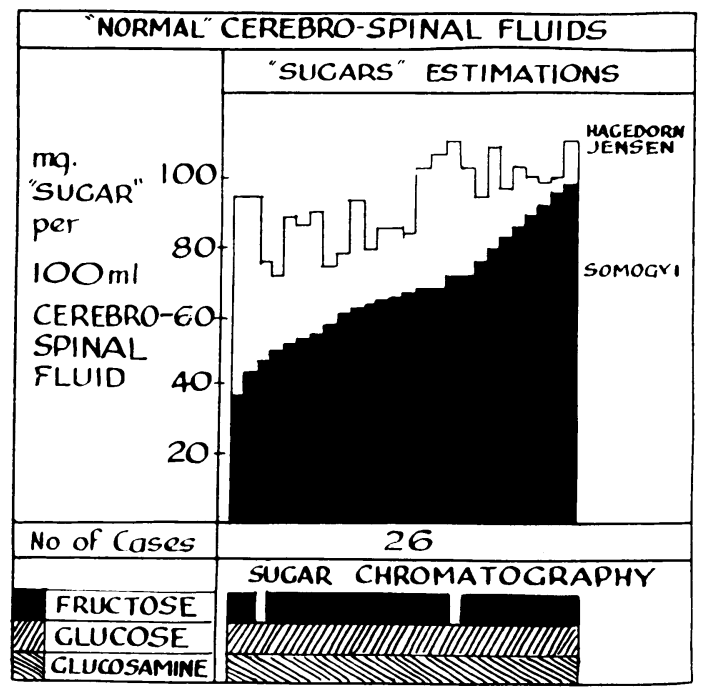

FIG. 1.-Comparison of normal cerebrospinal fluids by three methods of examination for reducing "sugars " presented in ascending order of Somogyi estimation. Sugars in chromatogram diagrams are represented as being normal, absent, or diminished considerably.

Hagedorn-Jensen figures vary between 66 and 112 $\mathrm{mg}$. with an average of $88 \mathrm{mg} . / 100 \mathrm{ml}$.

In the chromatograms three spots, glucosamine hydrochloride and base ( $\operatorname{Rf} 0.14-0.16)$, glucose ( $R f 0.18$ ), and fructose ( $R f 0.23$ ), are present in all but two of the samples, in which fructose was missing. (The standard glucosamine hydrochloride used for purposes of comparison also always produced two spots, hydrochloride and base in butanol acetic acid.)

No absolute relationship exists between the Somogyi and Hagedorn-Jensen figures. When the Somogyi estimations are arranged as in Fig. 1 in ascending order, the Hagedorn-Jensen figures are not in ascending order: the Hagedorn-Jensen method estimates other unsaturated compounds, for example, those containing double bonds, as well as sugars, while Somogyi claims that his method estimates most nearly the true sugar content. The figures in this group thus show an average of $25 \%$ non-sugars.

In a further 24 normal samples in which Hagedorn-Jensen determinations only were carried out the chromatographic results were similar.

In no case out of the total 50 was glucose absent and fructose present. In the six cases where no fructose appeared the disease diagnosis was diverse (including two cases of bronchopneumonia, one each of epilepsy, petit mal, tonsillitis, and convulsions).

When fructose was not apparent on the chromatogram, the fructose content by Roe's method was 
less than $1 \mathrm{mg} . / 100 \mathrm{ml}$. of cerebrospinal fluid. A very few specimens showed definite brown spots on the chromatograms at $\mathrm{Rf} 0.28$ (the $\mathrm{Rf}$ of xylose) and four specimens gave evidence of a keto form of pentose at the $\mathrm{Rf}$ of the urine sugar keto-xylose, 0.29 . In no specimen was a spot for reduced ascorbic acid noticed.

Group II.-Fig. 2 represents tuberculous meningitis cases in hospital. The 19 specimens from the 14 cases under treatment were taken in the resting periods between streptomycin treatment.

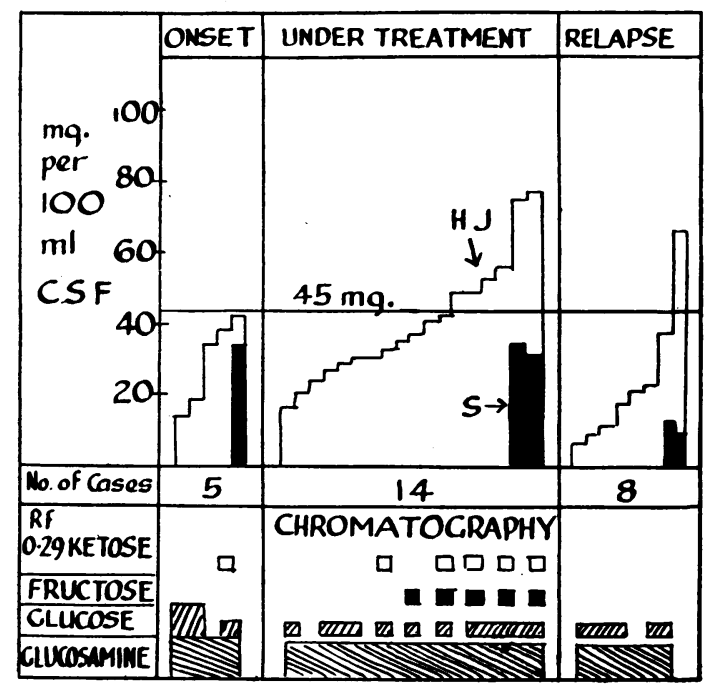

FIG. 2.-Comparison of tuberculous meningitis cerebrospinal fluids by three methods of examination for reducing sugars as in Fig. 1.

The Hagedorn-Jensen figures are arranged in ascending order. In this group they are mainly below $45 \mathrm{mg}$. while the few anomalous cases above $45 \mathrm{mg}$. gave Somogyi figures below 45 .

In the corresponding chromatograms much of the normal sugar is absent. From only five specimens traces of the fructose spot appeared; from all the others it was absent. Glucose spots were smaller and in certain early cases had disappeared completely.

In addition to the 27 tuberculous meningitis specimens charted in Fig. 2 a further 14 specimens not shown on Fig. 2 were examined: (1) seven " tests of cure," all cases with a cell count under 10 , and (2) seven cases of miliary tuberculosis with no meningeal involvement. In all cases the HagedornJensen figure was over $45 \mathrm{mg}$. and in all chromatograms all three normal spots were present. In only one case did the ketose ( $\operatorname{Rf} 0.29$ ) occur.
Fig. 3 represents the few non-tuberculous meningitis cases. Column 1 shows two cases of aseptic meningitis examined at the onset. For Case 1, first and sixth day specimens were examined and for Case 2, those of the first, second, third, fifth, and thirteenth days. (Penicillin was administered on the second.) Case 3 is of pneumococcal, Cases 4 and 5 of meningococcal, and Case 6 of benign lymphocytic meningitis.

Somogyi estimations in Cases 1 and 2 are well below $45 \mathrm{mg}$. until the thirteenth day (Case 2), whilst the Hagedorn-Jensen results are consider-

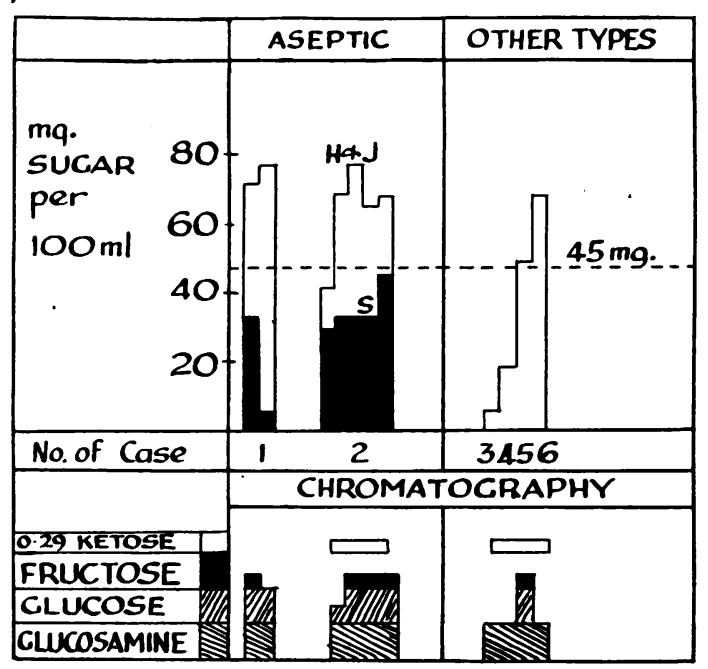

Fig. 3.-Comparison of cerebrospinal fluids of various types of non-tuberculous meningitis for reducing sugars by three methods as in Fig. 1 .

ably above $45 \mathrm{mg}$. except on the first day. The disappearance of fructose and decrease of glucose from the chromatogram is similar to that in tuberculous meningitis ; there is also an occasional trace of a second ketose.

Fructose Estimations (Roe Method).-Groups I and II are collected on one diagram (Fig. 4) for purposes of comparison.

Simultaneous chromatography and estimation of ketose by Roe's resorcinol method showed that the ketose estimated in the normal specimens was fructose. In the pathological specimens the method would estimate as fructose the trace of a second ketose which was present in three of the specimens in column 3 . Column 2 indicates that the normal range for fructose was 1.5 to $7.0 \mathrm{mg}$. The one exception was the lowest, a hypoglycaemia in which the blood sugars had fallen to $28 \mathrm{mg}$. In tuberculous meningitis (column 3) all fructose 


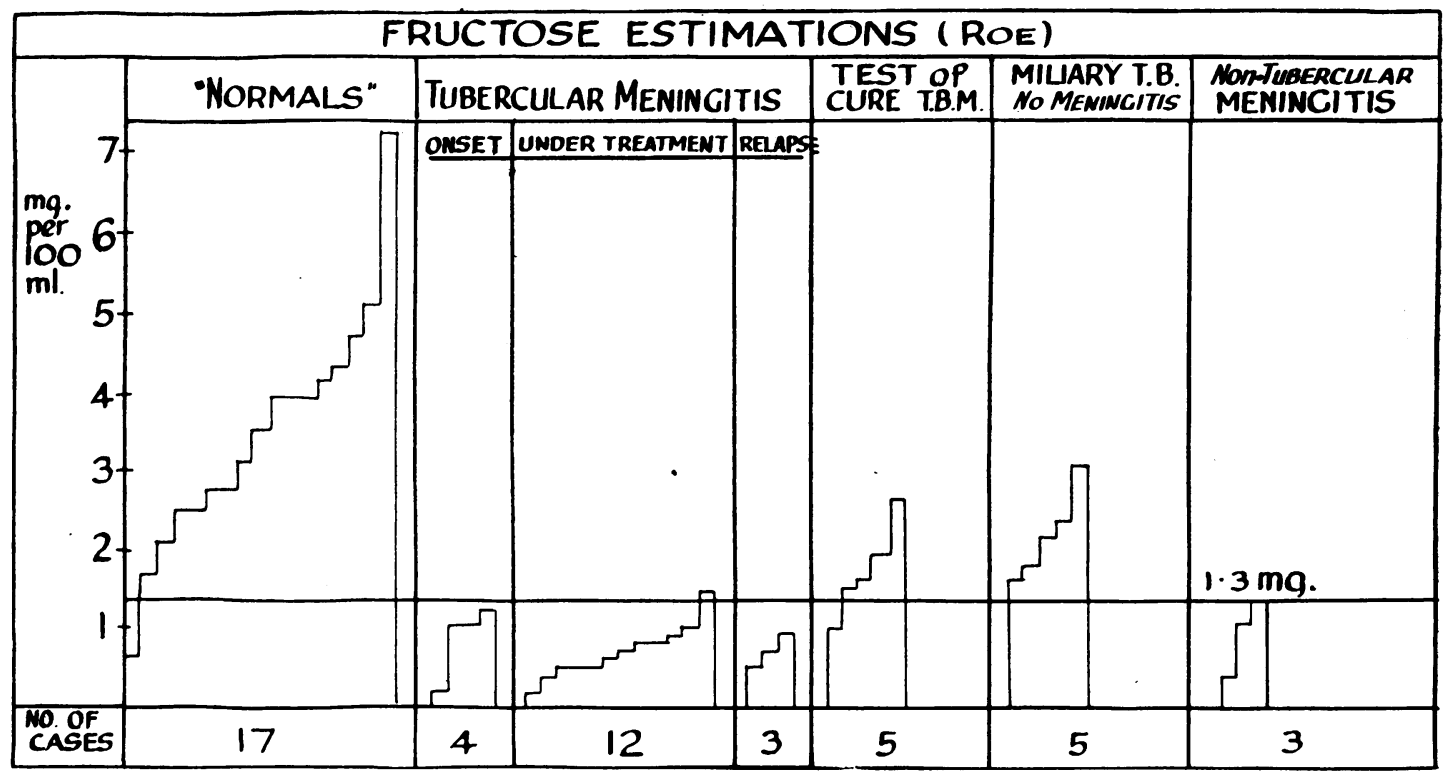

FIG. 4.-Comparison of fructose content of normal and tuberculous meningitis cerebrospinal fluid by Roe's method.

figures were below $1.5 \mathrm{mg}$. and in "onset" and "relapse" ranged from 0.2 to $1.0 \mathrm{mg} . / 100 \mathrm{ml}$.

In columns 6 and 7 the " tests of cure" of tuberculous meningitis and miliary tuberculosis cases, the fructose range is similar to the normal range and no second ketose was found. It seems fair to conclude that the ketose estimated by Hubbard and Russell (1951) was fructose in the normal material and that the occurrence of the second ketose is pathological and occasional.

TABLE I

HEXOSAMINE* ESTIMATION (MORGAN-ELSON METHOD)

\begin{tabular}{|c|c|c|}
\hline & & $\begin{array}{c}\text { Hexosamine } \\
\text { (mg. per } 100 \mathrm{ml} .)\end{array}$ \\
\hline $\begin{array}{l}\text { Range of normals ( } 10 \text { cases) } \\
\text { Range of tuberculous meningitis: } \\
\text { Under treatment ( } 10 \text { cases) } \\
\text { Yielding to treatment ( } 10 \text { cases) } \\
\text { Relapse ( } 3 \text { cases) }\end{array}$ & $\begin{array}{l}\cdots \\
\cdots \\
\cdots \\
.\end{array}$ & $\begin{array}{l}5-18(\text { mean } 9) \\
0-12(\text { mean } 7) \\
3-20(\text { mean } 9) \\
6-16(\text { mean } 10)\end{array}$ \\
\hline
\end{tabular}

In view of the criticism of Horowitz, Ikawa, and Fling (1950) of the effect of ketose and lysine in large quantities on the Morgan-Elson estimation the actual figures are not given too much weight except to emphasize that hexosamine estimation is of little value in the diagnosis or prognosis of meningitic disease.

In contrast to fructose, the hexosamine values obtained showed no great divergence between normal specimens and those from meningeal disease.
Effect of Antibiotics on the Chromatographic $\vec{\oplus}$ Method.-Owing to increased permeability of the $N$ blood brain barrier, levels of penicillin and strepto mycin detectable in the cerebrospinal fluid have been found in meningitis (Rosenberg and Sylvester; 1944). The possible effect of streptomycin on the chromatogram was therefore investigated.

It was found that streptomycin itself did not move forward in butanol acetic, whilst the acidhydrolysed products of streptomycin gave a brown sugar spot with benzidine at $\mathrm{Rf} 0.10$ and a reaction with the Morgan-Elson reagent at approximately the same Rf, probably the known acid hydrolysis product streptobiosamine. No spots within the range of a normal or tuberculous meningitis cerebrospinal fluid chromatogram were observed.

Streptomycin gave no positive reactions with the Roe colorimetric estimation for fructose, so this method might have advantages over other sugar estimation methods as an aid to prognosis in meningitis.

\section{Discussion}

Source of Glucosamine and Fructose.-Two sources of the hexosamine were thought possible: (1) that it might be combined in a polysaccharide or mucoprotein as in blood serum (Shetlar, 6 Foster, Kelly, and Everett, 1948) ; (2) that transamination from amino-acids on to the sugars was taking place.

To investigate the first supposition the precipitate from methanol extracts from the normal speci- 
mens was hydrolysed with $15 \% \mathrm{HCl}$ in sealed tubes for from three to six hours at $100^{\circ} \mathrm{C}$. Morgan-Elson estimations and chromatograms of such hydrolysates showed a hexosamine-reacting material at about $\mathrm{Rf} 0.08$ to 0.10 , but amounts were always less than $2-3 \mathrm{mg} . / 100 \mathrm{ml}$. cerebrospinal fluid.

It seems as though there is very little hexosamine containing polysaccharide normally in cerebrospinal fluid. Very enlarged glucosamine spots were found only in the cerebrospinal fluid of pneumonia cases and this may be connected with the already noted high glucosamine polysaccharide values of pneumonia blood sera (Nilsson, 1937). This observation is some slight evidence towards the hypothesis that the blood serum polysaccharide may be providing the hexosamine of the cerebrospinal fluid in an easily freed or free form.

The possibility of the second supposition being part of the truth is indicated by recent work on the chromatography of amino-acid and glucose or fructose mixtures. Obviously more work is required to determine the answer.

Arguing from the swift disappearance of fructose in meningeal infection, it would appear that an enzyme in the cells of the meninges might be responsible for the normal presence of fructose in cerebrospinal fluids. Although the brain selectively uses glucose rather than fructose (Maddock, Hawkins, and Holmes, 1939 ; Klein, 1944), the latter cannot reasonably all be supplied by the blood, as the fructose content of serum is so very slight and its rate of transfer from blood to brain five to 10 times less than glucose (Klein, Hurwitz, and Olsen, 1946). Furthermore, at least in vitro, Meningococcus and Myco. tuberculosis are not actively glycolytic (Hendry, 1939). It may be that glucosamine is the "easily changeable form of glucose" postulated by Hubbard and Russell as existing in cerebrospinal fluid. Again more work is required, particularly on a comparison between vascular, cisternal, and lumbar punctures. As a corollary to this study it has only been possible to compare a few cisternal and lumbar punctures by chromatography, when little or no difference was observed except in acute and old cases of hydrocephalus or meningitic blocks. No material has been chromatographed from the perivascular spaces of the brain, which might conceivably have a fructose content more nearly that of blood serum.

Estimation Methods.-The results obtained by the Hagedorn-Jensen and Somogyi methods of estimation of reducing substances support the work of Cacioppo and Bevilotti (1945), except that the ratio of the means is higher in this work. In meningitis it would appear that there is less likelihood of encountering the anomalous cases showing over $45 \mathrm{mg}$. when the Somogyi method is used.

\section{Summary}

The copper reduction methods of Somogyi give lower results than the Hagedorn-Jensen method in cerebrospinal fluid sugar estimations in both normal and meningitic cases.

Paper chromatography has demonstrated that there are at least three reducing substances present in cerebrospinal fluid from normal people and from cases of non-cerebrospinal disease. These substances are glucose, fructose, and glucosamine. In addition there are substances not yet identified, with a reducing action on both Hagedorn-Jensen and Somogyi reagents.

Fructose values fall at the onset of tuberculous meningitis, remain low during illness, regain low normality on recovery, and fall rapidly in relapse. Their estimation would appear to be of value in helping the rapid diagnosis of meningeal disease:

The range of hexosamine values does not appear to differ significantly in meningeal disease from that of normal values, at least by the MorganElson method of estimation.

We wish to thank Dr. R. P. Cook, of the Biochemical Department, University College, Dundee, where this work was carried out, and also the Bacteriology Department for specimens and information.

All tuberculous meningitis specimens in Fig. 4 and 27 of the 46 specimens in Group II were investigated with the co-operation and diagnostic assistance of Dr. W. M. Jamieson, Physician Superintendent, King's Cross Isolation Hospital, Dundee, and Dr. J. H. Prain, of the Pathology Department, Royal Infirmary, Dundee.

\section{REFERENCES}

Cacioppo, F. C., and Bevilotti, V. B. (1945). Boll. Soc. ital. Biol.

Cohen, H.(1936). Quart. J. Med. 5, 159

Flood, A. E., Hirst, E. L., and Jones, J. K. (1947). Nature, Lond., $160,86$.

Forsyth, W. G. C. (1948). Ibid., 161, 239

Harrison, G. A. (1947). Chemical Methods in Clinical Medicine, 3rd ed. London.

Hendry, E. (1939). Arch. Dis. Chi!d., 14, 307.

Horowitz, H. N., Ikawa, M., and F.ing, M. (1950). Arch. Biochem. $25,226$.

Horrocks, R. H. (1949). Nat:are, Lond. 164, 444.

Hubbard, R. S., and Russell, N. M. (1937). J. biol. Chem., Lond., $119,647$.

Jolles, Z. E., and Morgan, W. T. J. (1940). Biochem. J., 34, 1183.

Klein, J. R. (1944). J. biol. Chem., 153, 295.

- Hurwitz, R.. and Olsen, N. S. (1946). Ibid., 164, 509.

Maddock, S., Hawkins, J. F., and Holmes, E. (1939). Amer. J. Physiol., 125, 551.

MacCarthy, D., and Mann. T. P. (1950). Lancet, 1, 341.

Morgan, W. T. J., and Elson, L. A. (1934). Biochem. J., $28,988$.

Nilsson, I. (1937). Biochem. Z., 291, 254.

Partridge, S. M. (1948). Biochem. J., 42, 238

Roe, J. H. (1934). J. biol. Chem., 107, 15

Rosenberg, D. H., and Sylvester, J. C. (1944). Science, 100, 132

Shetlar, M. R., Foster. J. V., Kelly, K. H., and Everett, M. R. (1948). Proc. Soc. exp. Biol., N.Y., 67, 125.

Somogyi, M., and Kramer, H. V. (1928). J. biol. Chem., 80, 733. 